\title{
La théorie économique de l'auto-protection
}

\section{Monsieur Jean-Jacques Laffont}

\section{Résumé}

L'objet de cette note est de prendre en compte le phénomène d'autoprotection dans une théorie des assurances d'équilibre général. Après avoir mis en évidence la possibilité d'inexistence de l'équilibre concurrentiel et son inefficacité très générale, lorsqu'il existe, nous étudions des politiques économiques susceptibles de rétablir l'optimalité parétienne : prime variable liée à l'activité d'autoprotection, prime variable liée à la quantité d'assurance sélectionnée, prime définie sur la base des déclarations de l'agent et contrôlée par une cour de justice, etc.

\section{Abstract \\ The economic theory of self-protection}

The purpose of this paper is to take info account self'protection in a general equilibrium theory of Insurance. First, we show why the competitive equilibrium may fait to exist and why it is in general Pareto inefficient, when it cxists. Then, we study a number of economic policies designed to restore Pareto efficiency : Insurance premium variable with the level of self protection, with the quantity of Insurance, Insurance premium defined on the basis of the agent's declarations, etc.

Citer ce document / Cite this document :

Laffont Jean-Jacques. La théorie économique de l'auto-protection. In: Revue économique, volume 27, n4, 1976. pp. 561-588; http://www.persee.fr/doc/reco_0035-2764_1976_num_27_4_408274

Document généré le 28/05/2016 


\section{LA THEORIE ECONOMIQUE DE L'AUTO-PROTECTION 1}

\section{$\mathbf{L}$}

A THÉorie ÉCONOMrQue des assurances a tout d'abord pour objet d'analyser le comportement des compagnies d'assurance et le comportement des autres agents économiques face aux contrats proposés par les compagnies. Au-delà de cette étude microéconomique, il est important de se poser aussi des questions macroéconomiques. Est-ce que tel système d'assurances réalise une bonne allocation des risques? Est-ce que cette allocation est stable, au sens qu'elle n'incite pas l'entrée de nouveaux agents sur le marché des assurances? Est-elle optirnale au sens de Pareto? Sinon, pourquoi et quelles politiques économiques peuvent y remédier?

Si les études microéconomiques répondant aux problèmes que se posent les compagnies d'assurance dans la gestion de leurs affaires ont été nombreuses, les études d'équilibre général, qui ont un fort contenu normatif, sont beaucoup plus rares. Une raison importante de cette lacune est que l'introduction des assurances dans la théorie de l'équilibre général pose des problèmes nouveaux, liés en particulier à l'asymétrie « informationnelle » existant entre les compagnies d'assurance et leurs assurés; or, la théorie économique commence à peine à aborder les problèmes liés à l'information. La difficulté fondamentale est que les compagnies d'assurance ne connaissent pas parfaitement les risques qu'elles assurent et que les agents économiques, qui souvent les connaissent mieux que les compagnies, peuvent en outre les modifier par leur comportement.

Pour progresser dans la compréhension de ces phénomènes, il est bon de considérer des situations extrêmes, comme le cas où les agents

1. Une partie de cette note représente une révision d'un article commun avec E. HeLpMan [12]. Je tiens à remercier chaleureusement le Département de l'Education du Québec et le Département d'Economie de l'Université de Montréal qui en a permis la réalisation. Une version préliminairc A circulé comme Cahier $n^{\circ}$ 7503, Département de Sciences économiques, Université de Montréal 
sont différents quant à leurs risques, mais ne peuvent pas les changer, ou bien le cas où ils sont identiques quant aux risques mais différents quant à leurs préférences certaines, ou bien le cas où ils sont identiques mais se différencient par leur comportement, etc. Autant de sujets de recherche dont l'étude approfondie constitue les premiers pas dans la construction d'une théorie de l'équilibre général avec les compagnies d'assurance.

L'objectif de cette note est d'analyser en détail un des cas extrêmes mentionnés ci-dessus, en espérant susciter des recherches analogues pour avancer vers une synthèse qui prenne en compte simultanément les diverses difficultés. Le cas étudié ici correspond à une économie où tous les consommateurs sont identiques quant à leurs risques fondamentaux, et où ils peuvent par certaines de leurs activités influencer ces risques et éventuellement se différencier. Dans un section préliminaire, nous introduisons un vocabulaire qui nous sera utile pour présenter rapidement les travaux existants et pour définir plus précisément notre sujet.

\section{PRELIMINAIRES}

Afin d'introduire quelques définitions, considérons tout d'abord un modèle simplifié. Soit un agent économique qui peut se trouver dans deux états (de santé) à la fin de la période envisagée. Dans l'état 1 (bonne santé) il jouit d'un revenu $R$, alors que dans l'état 2 (mauvaise santé) son revenu n'est plus que $R-L$; $L$ représente la perte de revenu due à sa mauvaise santé. Au début de la période, il peut s'assurer et payer une prime $\mathrm{P}(z)$ pour recevoir $z$ au cas où il serait dans l'état 2. Soit II la probabilité qu'il soit dans l'état 1. Supposons de plus qu'il puisse, également au début de la période, influencer par l'achat de bien médical préventif $x_{0}$, la probabilité II d'être dans l'état 1 , ou (et) le montant de la perte L. Enfin, notons $x_{1}$ et $x_{2}$ les revenus consommés dans les états 1 et 2.

Si nous supposons que l'agent économique détermine son comportement par la maximation de l'espérance mathématique de son utilité, son programme s'écrit :

$$
\begin{array}{ll}
\operatorname{Max}\left\{\Pi\left(x_{0}\right) \mathrm{U}_{1}\left(x_{1}\right)+\left(1-\Pi\left(x_{0}\right)\right) \mathrm{U}_{2}\left(x_{2}\right)\right\} \\
\mathrm{T} . \mathrm{Q} . & x_{0}+x_{1}+\mathrm{P}(z) \leqq \mathrm{R} \\
& x_{0}+x_{2}+\mathrm{P}(z) \leqq \mathrm{R}-\mathrm{L}\left(x_{n}\right)+z \\
& x_{0}, x_{1}, x_{2}, z \geqq 0
\end{array}
$$


Nous dirons que $x_{0}$ représente une activité d'cuto-protection, Iorsque $x_{0}$ influence la probabilité $\Pi$.

Nous dirons que $x_{0}$ représente une activité d'auto-assurance, lorsque $x_{0}$ influence le montant de la perte $\mathrm{L}$.

La demande d'assurance par l'agent est la solution en $z$ du programme (1-1); elle devient une indemnité lorsque l'état 2 se réalise. La prime d'assurance qui lui correspond est alors $\mathbf{P}(z)$.

Le problème du risque moral («moral hazard») est l'effet de la disponibilité d'une assurance sur le montant d'auto-protection (ou d'autoassurance).

Nous dirons qu'il y a un problème de sélection adverse lorsque, en face d'un prix d'assurance unique, il existe des agents avec des risques différents et seuls les agents avec les plus gros risques s'assurent.

La théorie économique des assurances a été inexistante jusqu'à l'utilisation, pour formaliser le comportement de l'assuré, du modèle d'espérance mathématique devenu très courant dans la théorie de l'incertain. De nombreux articles, Arrow [3], Borch [6], Gould [10], Mossin [18], Pashigian et alii [19], Smith [24], etc., ont alors développé la théorie de la demande individuelle d'assurance et posé le problème d'un système d'assurance optimal. Arrow [5] a récemment généralisé un grand nombre de résultats au cas de fonctions d'utilité dépendant des états. Arrow [4] a introduit dans la littérature écono. mique le problème du risque moral défini ci-dessus, et a suscité une vive controverse sur la signification du phénomène (voir Pauly [20], Demsetz [8]). Ehrlich et Becker [9] ont considérablement clarifié le vocabulaire employé par les économistes, et étendu l'analyse de la demande au cas de l'auto-protection et de l'auto-assurance, tandis que Pauly [21], à la suite de Akerlof [1], a souligné le problème de sélection adverse, dû à des risques différents et non identifiables (voir aussi Rothschild et Stiglitz [23]).

Il est bien connu que le modèle d'équilibre général (Debreu [7], chap. 3-4-5) peut être réinterprété dans un environnement incertain, en associant à chaque état de la nature un marché pour chaque bien (Arrow [2], Debreu [7], chap. 6). Dans cette généralisation, les agents économiques ont des distributions de probabilité subjectives qui, avec leurs goûts, leurs ressources initiales et les prix, déterminent leur comportement. Sous les hypothèses classiques de convexité, l'équilibre concurrentiel est un optimum de Pareto. Radner [22] a bien souligné que la propriété d'efficacité de ces équilibres doit se comprendre comme efficacité par rapport à une structure d'information donnée, c'est-à-dire efficacité ex ante. 
La difficulté majeure de la solution Arrow-Debreu est le nombre prohibitif de marchés qu'elle requiert. Une tâche fondamentale de la théorie économique est donc l'étude d'économies pourvues d'un nombre restreint de marchés. Malinvaud [15] [18] a montré comment, avec des risques individuels, une économie décentralisée pouvait réaliser un optimum, avec seulement des marchés d'assurance. Dans cette note, nous présentons une analyse d'équilibre général du phénomène d’autoprotection (pour un modèle sans marchés futurs mais avec des marchés d'assurance) qui révèle plusieurs difficultés passées inaperçues dans l'analyse partielle contenue dans les contributions citées ci-dessus. Notre problème peut s'interpréter comme un cas particulier du problème général défini ci-dessus. Dans le modèle (1.1), il existe un seul marché d'assurances alors qu'il faudrait un marché pour chaque valeur de $x_{0}$, pour retrouver le modèle étudié par Malinvaud.

Dans la section 3, nous montrons comment l'auto-protection peut affecter l'existence de l'équilibre, c'est-à-dire le test le plus simple de cohérence logique du modèle. La section 4 explique pourquoi, en présence d'auto-protection, l'équilibre concurrentiel avec primes constantes est en général inefficace. Deux solutions sont envisagées dans la section 5, une prime variable et la taxation. Enfin, des exemples de solution de second rang sont développés dans la section 6 .

\section{DESCRIPTION DE L'ECONOMIE}

Par souci de simplicité, nous considérons une économie d'échange dans laquelle consommateurs et compagnies d'assurance sont les seuls agents économiques.

\section{A. Les consommateurs}

Les consommateurs sont tous identiques et vivent deux périodes. La population croît au taux uniforme $\rho$. A chaque instant $t$, il y a des jeunes et des vieux consommateurs, et les jeunes sont $(\boldsymbol{1}+\rho)$ fois plus nombreux que les vieux. Soit $\mathrm{N}_{t}$, le nombre de consommateurs à la date $t$.

Nous supposerons aussi qu'il n'existe qu'un seul bien de consommation. La généralisation du modèle à plusieurs biens et plusieurs types identifiables de consommateurs est immédiate. 
Dans la première période de leur vie, les consommateurs ont une ressource initiale certaine $w_{0}$. Par contre, ils peuvent se trouver dans l'un des $S$ états (de santé) possibles pendant la deuxième période. Soit $w_{s}, s=1, \ldots, S$, la ressource initiale dans l'état $s$. En plus de procurer (éventuellement) des satisfactions, la consommation, pendant la première période, $x_{0}$, peut influencer les probabilités $\Pi_{s}, s=1, \ldots, \mathrm{S}$ des différents états. Par conséquent, $\Pi=\Pi_{1}, \ldots, \Pi_{\mathrm{s}}$ ) est une fonction définie sur $R_{+}$à valeurs dans le simplexe de $R^{s}$. Le vecteur de consommation d'un agent type est noté : $\boldsymbol{x}=\left(x_{0}, x_{1}, \ldots, x_{\mathrm{s}}\right)$ où $x_{s} \in \mathrm{R}, s=1, \ldots, \mathrm{S}$ est la consommation dans l'état $s$ de la deuxième période.

Hypothèse 1. $w_{s} \supseteq 0, s=0,1, \ldots, \mathrm{S}$ avec au moins une inégalité stricte. Pour chaque consommateur, l'ensemble de consommation est dans chaque période $R_{+}$.

Il n'existe pas de marchés futurs, mais, dans la première période de sa vie, un consommateur peut s'assurer contre chaque état $s=1, \ldots, S$. Au même prix ${ }^{2}$, il peut auıssi emprunter (s'assurer négativement) dans la première période avec obligation de remboursement dans la seconde période s'il est dans un certain état $s=1, \ldots, S$. Ces transactions sont effectuées avec des compagnies d'assurance.

Soit $\boldsymbol{z}_{s}$ la quantité d'assurance contre l'état $s$ achetée par un consommateur-type, soit $q_{s}$ le prix d'une unité d'assurance contre l'état $s$ ( $z_{s}$ peut être positif ou négatif) ${ }^{3}$. Notons $z=\left(z_{1}, \ldots, z_{\mathrm{s}}\right)$ et $q=\left(q_{1}, \ldots, q_{s}\right)$.

Hypotrèse 2. Les préférences du consommateur-type sont représentables par une fonction d'utilité $V(x)$ telle que :

$$
V(x)=\sum_{s=1}^{S} \Pi_{s}\left(x_{0}\right) \cdot U_{s}\left(x_{0}, x_{s}\right)
$$

où $\Pi_{s}($.$) et U_{s}(.,$.$) sont des fonctions continues pour s=1, \ldots, S$. De plus $U_{s}(. .$.$) est croissante en x_{s}$, pour au moins un $s^{*} \in\{1, \ldots, S\}$.

2. L'hypothèse d'un seul prix pour des assurances positives ct négatives doit être justifiée. Dans une analyse d'équilibre temporaire où les banqueroutes sont possibles, il faudrait tenir compte des risques de défaut et il existe une dissymétrie évidente entre un consommateur et une compagnie d'assurance qui, elle, bénéficie de la loi des grands nombres.

3. Un modèle plus réaliste mais plus complexe comporterait un marché de la monnaie où il est possibie de s'endetter et des marchés d'assurances sur lesquels on ne peut s'assurer que positivement. Les résultats obtenus dans un tel modèle sont toutefois analogues à ceux présentés ici (voir Laffont [13]). 
Nous pouvons maintenant formuler le programme d'optimation du jeune consommateur-type :

$$
\begin{aligned}
& \operatorname{Max} \mathrm{V}(x) \\
& \text { T.Q. } x_{0} \leqq w_{0}-\sum_{s=1}^{S} q_{s} z_{s} \\
& x_{s} \leqq w_{s}+z_{8} \quad s=1, \ldots, \mathrm{S} \\
& x_{s} \geqq 0 \quad s=0, \ldots, S
\end{aligned}
$$

Les contraintes de ce programme peuvent être récrites :

$$
\begin{gathered}
x_{0}+\sum_{s=1}^{\mathrm{S}} a_{s} x_{s} \leqq w_{0}+\sum_{s=1}^{\mathrm{S}} q_{s} w_{s} \\
x_{s} \geqq 0 \quad s=0,1, \ldots, \mathrm{S} \text { avec } z_{s}=x_{s}-w_{s}
\end{gathered}
$$

Comme nous nous intéressons seulement aux équilibres stationnaires, c'est-à-dire aux équilibres pour lesquels les prix et quantités consommées sont constants au cours du temps, le comportement des consommateurs âgés se déduit également du programme ci-dessus.

Il est clair que pour pouvoir parler rigoureusement d'état stationnaire, il faut un nombre infini de consommateurs, de sorte que les fréquences s'identifient avec les probabilités et l'incertitude disparaisse au plan macroéconomique. Nous utiliserons dans notre modèle cette approximation. Une formalisation rigoureuse qui ne change rien ci-dessous est obtenue en représentant l'ensemble des agents par un continuum $[0,1]$.

\section{B. Compagnies d'assurance}

Il existe des entreprises, appelées compagnies d'assurance, qui, au prix $q_{s}, s=1, \ldots, \mathrm{S}$, acceptent de satisfaire les demandes d'assurance des consommateurs décrites ci-dessus. La transaction est telle que la compagnie d'assurance reçoit d'un jeune consommateur $q_{s} z_{s}$ (ou lui prête) $q_{s} z_{s}$ si $z_{s}$ est négatif et lui paie (ou reçoit de lui) $z_{s}$, s'il est dans l'état $s$ lorsqu'il est âgé.

Nous serons amenés à faire différentes hypothèses sur les compagnies d'assurance.

HYPothèse 3. Il y a concurrence parfaite entre les compagnies d'assurance 4 .

4. Dans cette hypothèse, nous requérons davantage que ce que l'on entend ustellement par concurrence parfaite. Plus précisément, lersque le nombre de consommateurs tend vers l'infini, nous voulons que le nombre de consommateurs assurés par chaque compagnie sur chaque type de contrat tende aussi vers l'infiri (ou soit zéro pour toutes les compagnies). 
Hypothèse $3^{\prime}$. Il existe une seule compagnie d'assurance avec une contrainte institutionnelle de profit nul sur chaque type de contrat, c'est-à-dire chaque $s=1, \ldots, \mathrm{S}$.

HYPOTHÈse $3 "$. Il existe une seule compagnie d'assurance avec une contrainte institutionnelle de profit global nul.

L'existence de compagnies d'assurance permet aux consommateurs de transférer conditionnellement du pouvoir d'achat de la période 1 à la période 2 de leur vie, et vice-versa. Des marchés futurs conditionnels pourraient procurer les mêmes possibilités, mais la structure des générations est telle que ces marchés ne peuvent apparaître ${ }^{5}$.

Le modèle de l'économie étudiée étant bien défini, nous pouvons aborder le premier test de cohérence logique d'un modèle, à savoir la question de l'existence d'un équilibre. Il est intéressant d'observer que, malgré l'existence d'un continuum d'agents, la présence de non-convexités dans les fonctions d'utilité peut conduire à l'inexistence de l'équilibre.

\section{EXISTENCE D'UN EQQUILIBRE}

Lorsqu'il existe plusieurs comportements, solutions du programme (2.1), nous permettons différents comportements pour des consommateurs identiques, de manière à donner la plus grande flexibilité possible au concept d'équilibre. Soit $\lambda_{a}$ la proportion de consommateurs qui choisissent le vecteur de consommation $x^{a}$.

Soit $\mathrm{Q}$, l'espace des prix : $\mathrm{Q}=\left\{q / q \in \mathrm{R}^{\mathrm{s}+1}, q_{0}=1, q_{s} \geqq 0\right.$, $s=1, \ldots, \mathrm{S}\}$.

DÉfrnitron. Un équilibre fort est un ensemble fini $A$, un ensemble de poids positifs $\lambda_{a}, a \in A$, tels que $\underset{a \in A}{ } \lambda_{a}=1$, un vecteur de prix $\bar{q} \in \mathrm{Q}$, un ensemble de vecteurs de consommation $\bar{x}^{a}=\left(x^{a}, \ldots, x^{a} \mathbf{s}\right)$, $a \in \mathrm{A}$, tels que :

5. Une sclution partielle serait la création de marchís futurs entre jeunes conscmmateurs. Ces marchés perinettraient de réaliscr un niveau d'utilité sûr pour la deuxième póriodé, mais ne permettraient pas de transferts de ressources entre les piriodes. 


$$
\begin{array}{r}
(1+\rho) \sum_{a \in \mathrm{A}} \lambda_{a} \bar{q}^{s}\left(\bar{x}_{s}^{a}-w_{s}\right)-\sum_{x \in \mathrm{A}} \lambda_{a} \Pi_{s}\left(\bar{x}_{0}^{a}\right)\left(\bar{x}_{s}^{a}-w_{s}\right)=0 \\
\text { pour } s=1, \ldots, \mathrm{S} \\
(1+\rho) \sum_{a \in \mathrm{A}} \lambda_{a}\left(\bar{x}^{a}{ }_{0}-w_{0}\right)+\sum_{a \in \mathrm{A}} \lambda_{a} \sum_{s=1}^{S} \Pi_{s}\left(\bar{x}^{a}{ }_{0}\right)\left(\bar{x}^{a}{ }_{s}-w_{s}\right)=0 \\
\bar{x}^{a}=\left(\bar{x}_{0}^{a}, \bar{x}^{a}{ }_{1}, \ldots, \bar{x}^{a} \mathrm{~S}\right) \in \xi(q)=\left\{x^{*} / x^{*} \text { est solution de }(2.1)\right\} \\
\text { pour tout } a \in \mathrm{A}
\end{array}
$$

La condition (3.1) exprime la nullité du profit avec probabilité un, sur chaque contrat $s=1, \ldots, \mathrm{S}$.

La condition (3.2) assure l'équilibre sur le marché du bien.

Enfin (3.3) dit que les consommateurs sont en équilibre.

Dans la définition d'un équilibre faible, la condition (3.1) est remplacée par :

$$
\begin{gathered}
(1+p) \sum_{s=1}^{s} \sum_{a \in \mathrm{A}} \lambda_{a} \bar{q}_{s}\left(\bar{x}_{0}^{a}-w_{s}\right) \\
-\sum_{s=1}^{s} \sum_{a \in \mathrm{A}} \lambda_{a} \Pi_{s}\left(\bar{x}_{0}^{a}\right)\left(\bar{x}_{s}^{a}-w_{s}\right)=0
\end{gathered}
$$

Si A est réduit à un seul élément, tous les consommateurs ont le même comportement. Nous dirons que de tels équilibres sont uniformes.

L'équilibre uniforme fort est particulièrement intéressant, car il permet une concurrence totale entre les compagnies d'assurance. Si un équilibre n'est pas uniforme, on peut montrer (voir Rothschild et Stiglitz [23]) que l'équilibre peut être détruit si les compagnies se font concurrence sur des contrats prix-quantité 6 . De plus, si l'équilibre est faible (et non uniforme), les compagnies d'assurance peuvent facilement choisir le meilleur type de contrat pour détruire l'équilibre.

Ainsi, il apparaît que le concept d'équilibre uniforme est compatible avec la concurrence (hypothèse 3), alors que l'équilibre faible nécessite l'existence d'une seule compagnie d'assurance.

Pour démontrer l'existence d'un équilibre uniforme fort, il est nécessaire de faire deux hypothèses supplémentaires.

6. En effet, en raison de la multiplicité de comportements possibles pour des consommateurs identiques, il peut se poser à l'équilibre un problème de sélection adverse, puisque des consommations différentes de bien préventif conduisent à des risques différents. Toutefois, par hypothèse, les compagnies d'assurance ne peuvent pas observer ces risques. Notons néanmoins que les consommateurs sont amenés à acheter đes quantités différentes d'assurance et que les agents qui ont ies plus faibles risques ont une incitation à signaler leur différence. 
HYPOTHÈsE 3. $\quad \underset{x>0}{\operatorname{Min} \Pi_{s}}(x) \geqq \Pi>0$ pour $s=1, \ldots, \mathrm{S}$.

Soit $I=\left[\frac{\Pi}{1+\rho}, 1-\Pi\right]$

Cette hypothèse est une façon simple d'éviter des problèmes techniques du type du paradoxe de St-Petersbourg.

Hypothèse 4. $V(x)$ est quasi concave.

Il est remarquable qu'une telle hypothèse soit nécessaire malgré le nombre infini de consommateurs. La nécessité de $\mathrm{H} 4$ est démontrée par la proposition 2, ci-dessous.

Proposition 1. Sous $\mathrm{H} 1$ à $\mathrm{H} 4$, il existe un équilibre uniforme fort.

Démonstration. Nous pouvons restreindre le vecteur-prix à l'ensemble compact $I^{\text {s }}$. Puisque $V(x)$ est quasi concave, sous $\cdot \mathrm{H} 1-\mathrm{H} 2$, la correspondance de demande d'un consommateur-type $\xi(q)$ de $I^{\text {s }}$ dans $\mathbf{R}_{+}^{\mathbf{s}+1}$ est à valeurs convexes compactes non vides et semi continue supérieurement (s.c.s.) (voir Debreu [7]). Son domaine est inclus dans un ensemble convexe et compact $\mathrm{C}$.

$\frac{\Pi(x)}{1+\rho}$ est une fonction continue de $C$ dans $I^{8}$.

Soit $\Psi$, la correspondance s.c.s. à valeurs convexes compactes non vides de l'ensemble convexe compact $\mathrm{C} \times \mathrm{I}^{\mathrm{s}}$ dans lui-même définie comme :

$$
\Psi(x, q)=\left[\xi(q), \frac{\Pi(x)}{1+p}\right]
$$

Par le théorème de Kakutani, elle a un point fixe :

$$
q^{*}=\frac{1}{1+\rho} \text { II }\left(x^{*}\right) \quad \text { pour } x^{*} \in \xi\left(q^{*}\right)
$$

Par conséquent, les conditions (2.1) et (2.3) de la définition d'un équilibre fort sont vérifiées. D'après $\mathrm{H} 2$, la loi de Walras est satisfaite pour $\left(x^{*}, q^{*}\right)$ de sorte que la condition (2.2) est également remplie.

Q.E.D.

Il est clair qu'un équilibre (uniforme) fort est aussi un équilibre (uniforme) faible.

Proposrtron 2. Sous $\mathrm{H} 1$ à $\mathrm{H} 3$, il peut ne pas exister d'équilibre ni fort, ni faible. 
Démonstration. Nous donnons un exemple, où $\mathrm{V}(x)$ n'est pas quasi concave, pour lequel il n'existe pas d'équilibre.

Soit, $\rho=0, \mathrm{~S}=2, w_{0}=10, w_{1}>0, w_{2}>0$.

$$
\begin{array}{ll}
\mathrm{U}_{1}\left(x_{0}, x_{1}\right)=x_{0}+10 & \\
\mathrm{U}_{2}\left(x_{0}, x_{2}\right)=x_{0} & \\
\mathrm{II}_{1}\left(x_{0}\right)=0.9-0.1 x_{0} & \text { si } 0 \leqq x_{0} \leqq 8 \\
=0.1 & \text { si } 8 \leqq x_{0}
\end{array}
$$

Le programme d'un consommateur-type peut alors s'écrire :

$$
\begin{array}{ll}
\text { Max } & x_{0}+10 \Pi_{1}\left(x_{0}\right) \cdot x_{1} \\
\text { T.Q. } & x_{0}+q_{1} x_{1}+q_{2} x_{2} \leqq 10+q_{1} w_{1}+q_{2} w_{2} \\
& x_{0}, x_{1}, x_{2} \geqq 0
\end{array}
$$

Nous avons représenté sur la figure 1 une carte d'indifférence. Il est clair que la solution en $x_{2}$ de $(3.5), x_{2}$, est nulle. De plus :

$$
\begin{aligned}
& \text { Pour } q_{1}<1 / 9, x_{0}^{*}=0 \\
& \text { Pour } q_{1}>1 / 9, x^{*}=10+q_{1} w_{1}+w_{2} w_{2}=\frac{10+q_{1} w_{1}+q_{2} w_{2}}{q_{1}} x_{1}^{*}=0
\end{aligned}
$$

$q_{1}<1 / 9$ ne peut pas correspondre à un équilibre puisque $x *_{0}=0$ implique $\Pi_{1}\left(x{ }_{0}\right)=0.9>q_{1}$, et le contrat d'assurance relatif à l'état 1 est actif puisque :

$$
z_{1}^{*}=x_{1}^{*}-w_{1}=\frac{10+q_{2} w_{2}}{q_{1}}>0
$$

De même $q_{1}>1 / 9$. correspond à $\Pi_{1}\left(x_{0}\right)=0.1<q_{1}$ et le contrat est actif puisque $z *_{a}=x *_{1}-w_{1}=-w_{1}<0$. Le seul équilibre possible est donc un équilibre non uniforme avec $q_{1}=1 / 9$. Les agents sont alloués en proportion $\lambda,(0<\lambda<1)$, au vecteur $\left(\boldsymbol{x}_{0}, \boldsymbol{x}_{1}{ }_{1}\right)=\left(0, \frac{10+q_{1} w_{1}+q_{2} w_{2}}{q_{1}}\right)$ et en proportion $1-\lambda$ au vecteur $\left(x_{0}^{b}, x_{1}^{b}\right)=\left(10+q_{1} w_{1}+q_{2} w_{2}, 0\right)$. L'équilibre sur le second contrat requiert :

$$
\mathrm{P}_{2}=\lambda\left[q_{2}-\Pi_{2}\left(x_{0}^{a}\right)\right]\left(x^{a}-w_{2}\right)+(1-\lambda)\left[q_{2}-\Pi_{2}\left(x_{0}^{b}\right)\right]\left(x_{2}^{b}-w_{2}\right)=0
$$

ou

$$
q_{2}=0.9-0.8 \lambda
$$

De même l'équilibre sur le premier contrat requiert : 


$$
P_{1}=\lambda\left[q_{1}-\Pi_{1}\left(x_{n}^{a}\right)\right]\left(x^{a}{ }_{1}-w_{1}\right)+(1-\lambda)\left[q_{1}-\Pi_{1}\left(x_{0}^{b}\right)\right]\left(x^{b}-w_{1}\right)=0
$$

ou

$$
639 \lambda\left(10+q_{2} w_{2}\right)+(1-\lambda) w_{1}=0
$$

qui nécessite $\lambda<0$ ou $\lambda>1$.

Il n'existe donc pas d'équilibre fort. Existe-t-il des équilibres faibles, c'est-à-dire tels que $\mathrm{P}_{1}+\mathrm{P}_{2}=0$ ? $\mathrm{P}_{1}+\mathrm{P}_{2}=0$ est équivalent à :

$$
\lambda=\frac{90\left(-q_{2} w_{2}+0.9 w_{2}\right)-w_{1}}{639 q_{2} w_{2}+72 w_{2}-w_{1}+6390}
$$

Selon les valeurs de $w_{1}$ et $w_{2}$ il existe ou non un équilibre faible.

Par exemple, si $w_{2}=90$ et $w_{1}=80, q_{1}=1 / 9, q_{2}=8 / 9, \lambda=1 / 6398$ est un équilibre.

Par contre, si $6390>w_{1}>90 w_{2}$, il n'existe pas de valeur de $q_{2}$ positive qui soit compatible avec $0<\lambda<1$.

Q.E.D.

L'inexistence d'un équilibre dans l'exemple numérique peut s'expliquer ainsi : quand le prix de l'assurance pour l'état 1 est inférieur à $1 / 9$, les agents achètent une quantité positive d'assurance, mais n'exercent aucune auto-protection. La probabilité d'être dans l'état 1 est par conséquent élevée et la compagnie d'assurance fait des pertes. Quand le prix de l'assurance devient supérieur à $1 / 9$, la non-convexité des préférences cause une modification complète du comportement des agents ; ils achètent une quantité négative d'assurance et un maximum d'auto-protection, ce qui, d'une façon différente, inflige des pertes à la compagnie d'assurance. Quand le prix de l'assurance est exactement $1 / 9$, les agents sont indifférents entre les deux comportements, mais comme la compagnie fait des pertes dans les deux cas, elle fait aussi des pertes pour toute combinaison des deux comportements.

Il est bon de s'interroger sur la signification d'un résultat d'inexistence d'équilibre. Souvent, un tel résultat est la justification a contrario d'une institution économique qui apparaît pour secourir le marché. Qu'en est-il ici ? Sans entrer dans les dótails, pour lesquels le lecteur peut se reporter à Laffont [14], disons que l'inexistence d'équilibre. ne semble pas poser de difficulté fondamentale à la viabilité du modèle. En effet, il est possible de le modifier quelque peu, pour aboutir à un modèle dans lequel l'absence d'un seul marché d'assurance (que l'on peut interpréter comme correspondant à l'état de l'économie pour lequel il n'y a pas d'accident) permet de démontrer l'existence d'un 


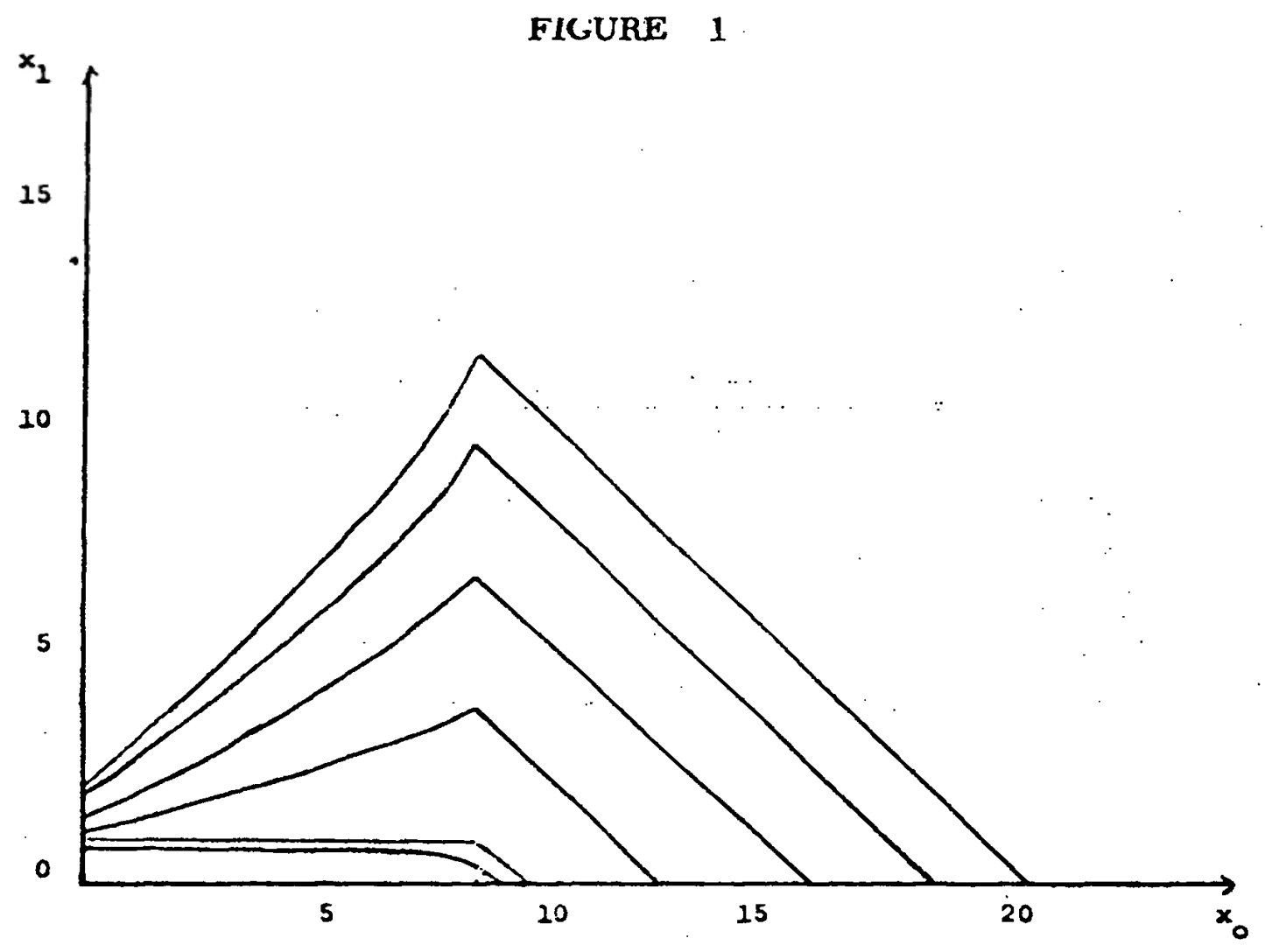

équilibre non uniforme. Dans l'exemple ci-dessus, cette hypothèse revient à supprimer la possibilité de s'assurer négativement dans l'étai 1. Cette hypothèse permet d'obtenir l'existence d'un équilibre, bien que l'optimum contraint par l'absence de ce «marché » reste optimum de Pareto.

La non-uniformité de l'équilibre pose toutefois des problèmes d'existence d'une autre nature (voir note de bas de page $n^{\circ} 6$ et RothschildStiglitz [23]).

\section{INEFFICACITE DE L'EQUILIBRE}

Nous ignorons dans cette section le problème d'existence (en supposant par exemple que $\mathrm{V}(x)$ est quasi concave) et nous étudions les propriétés des équilibres lorsqu'ils existent. En particulier, nous montrons que, de façon très générale, les équilibres concurrentiels sont inefficaces au sens de Pareto.

Après avoir donné un exemple d'équilibre concurrentiel inefficace. 
nous montrons sous des hypothèses de différentiabilité que l'équilibre est localement inefficace. Enfin, nous donnons une interprétation du résultat en termes d'effets externes.

Défintron. $\left[\mathrm{A},\left(\lambda_{a}\right),\left(\boldsymbol{x}^{a}\right)\right]$ est une allocation réalisable si :

(4.1) A est un ensemble fini et $A=\{$ union de tous les $a\}$.

(4.2) $\lambda_{a}>0$ pour tout $a \in \mathrm{A}$ et $\sum_{a \in \mathrm{A}} \lambda_{a}=1$.

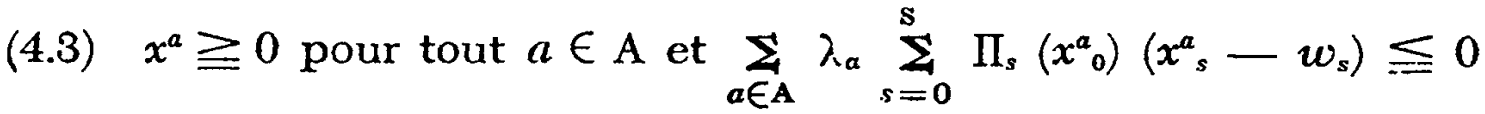
avec la convention $\Pi_{0}\left(x_{0}^{a}\right)=1+\rho$.

DÉfinttron. [A*, $\left.\left(\lambda_{a *}\right),\left(x^{a *}\right)\right]$ est une allocation efficace si :

(4.4) $\left[A^{*},\left(\lambda_{a *}\right),\left(x^{a *}\right)\right]$ est une allocation réalisable.

(4.5) Il n'existe pas d'autre allocation réalisable $\left[\mathrm{A},\left(\lambda_{\mathrm{a}}\right),\left(x^{a}\right)\right]$ telle que $A$ puisse être divisé en \# $A^{*}$ sous-ensembles disjoints $A^{a^{*}}, a^{*} \in A^{*}$, tels que :

(1) $\underset{a \in \mathrm{A}^{a *}}{\sum} \lambda_{a}=\lambda_{a *}$ pour tout $a^{*} \in \mathrm{A}^{*}$.

(2) $\mathrm{V}\left(x^{a}\right) \geqq \mathrm{V}\left(x^{a *}\right)$ pour tout $a^{*} \in \mathrm{A}^{*}, a \in \mathrm{A}^{a *}$, avec une inégalité stricte pour au moins un couple $\left(a^{\prime}, a^{* \prime}\right), a^{* \prime} \in \mathrm{A}^{*}, a^{\prime} \in \mathrm{A}^{a^{* \prime}}$.

Propositron 4.1. Sous HI-H4, un équilibre peut ne pas être efficace.

Démonstration. Nous construisons un exemple d'un équilibre uniforme fort qui est inefficace.

Soit

$$
\begin{gathered}
P=0, \mathrm{~S}=2, w_{0}=w_{1}=w_{2}=10 \\
\Pi_{1}\left(x_{0}\right)=0.9-0.8 \epsilon^{-x_{0}} \\
\mathrm{U}_{1}\left(x_{0}, x_{1}\right)=x_{1} \\
\mathrm{U}_{2}\left(x_{0}, x_{1}\right)=0
\end{gathered}
$$

L'optimum de Pareto est solution du programme suivant :

$$
\begin{array}{ll}
\text { Max } & x_{1}\left(0.9-0.8 \epsilon-x_{0}\right) \\
\text { T.Q. } & x_{0}+\left(0.9-0.8 \epsilon-x_{0}\right) \cdot x_{1}=20 \\
& x_{0}, x_{1} \geq 0
\end{array}
$$


La solution est évidemment $x_{0}=0, x_{1}=200$, soit un niveau d'utilité $\mathrm{V}^{0}=20$.

L'allocation d'équilibre est obtenue de la façon suivante : le programme d'un consommateur est défini par

$$
\begin{array}{ll}
\text { Max } & x_{1}\left(0.9-0.8 \epsilon-x_{0}\right) \\
\text { T.Q. } & x_{0}+q_{1} x_{1}=20
\end{array}
$$

Les conditions du premier ordre sont :

$$
\begin{gathered}
\left(0.9-0.8 \epsilon-x_{0}\right)=\lambda a_{1} \\
0.8 x_{1}, \epsilon^{-x_{0}}=\lambda
\end{gathered}
$$

L'équilibre de la compagnie d'assurance nécessite :

$$
q_{1}=\Pi_{1}\left(x_{0}\right) \text {, soit } \lambda=1 \text {, ou encore } x_{1}=\frac{\epsilon x_{0}}{0.8}
$$

Soit $x *_{0}$ la solution (unique et positive) de l'équation budgétaire :

$$
x_{0}+\left(0.9-0.8 \epsilon-x_{0}\right) \cdot \frac{\epsilon x_{0}}{0.8}=20
$$

L'équilibre correspond à :

$$
\begin{gathered}
q_{1}=0.9-0.8 \epsilon-x *_{0} \\
x_{0}=x_{0}^{*} \\
x_{1}=\frac{\epsilon *_{0}}{0.8}
\end{gathered}
$$

et au niveau d'utilité :

$$
\mathrm{V}^{\mathrm{x}}=20-x^{*}
$$

Q.E.D.

Sur cet exemple, il apparaît clairement que le comportement décentralisé conduit à une auto-protection qui est un gaspillage sur le plan collectif.

En dehors de la taxation ou de la prime variable discutée dans la suite de la section, il apparaît dans le cas de l'exemple qu'une solution simple est de supprimer le marché pour le bien préventif. On se trouve donc dans un cas où avec un ensemble d'institutions restreint, l'équilibre concurrentiel èst préférable (voir Green-Sheshinski [11], pour un exemple analogue).

Ce résultat trouve son interprétation générale dans la théorie du second rang (Je suis redevable à Roger Guesnerie d'une discussion 
éclairante sur ce point). Une organisation particulière (à la Arrow. Debreu) qui permettrait d'obtenir un optimum de Pareto impliquerait des marchés d'assurance pour chaque valeur de $x_{0}$. Nous pouvons considérer que nous avons supprimé un certain nombre de marchés Arrow-Debreu. Nous sommes donc dans un cadre de second rang. Il est alors clair que la suppression d'un marché supplémentaire conduit à un équilibre qui en général n'est pas comparable, au sens de Pareto, à l'équilibre précédent et peut éventuellement être meilleur.

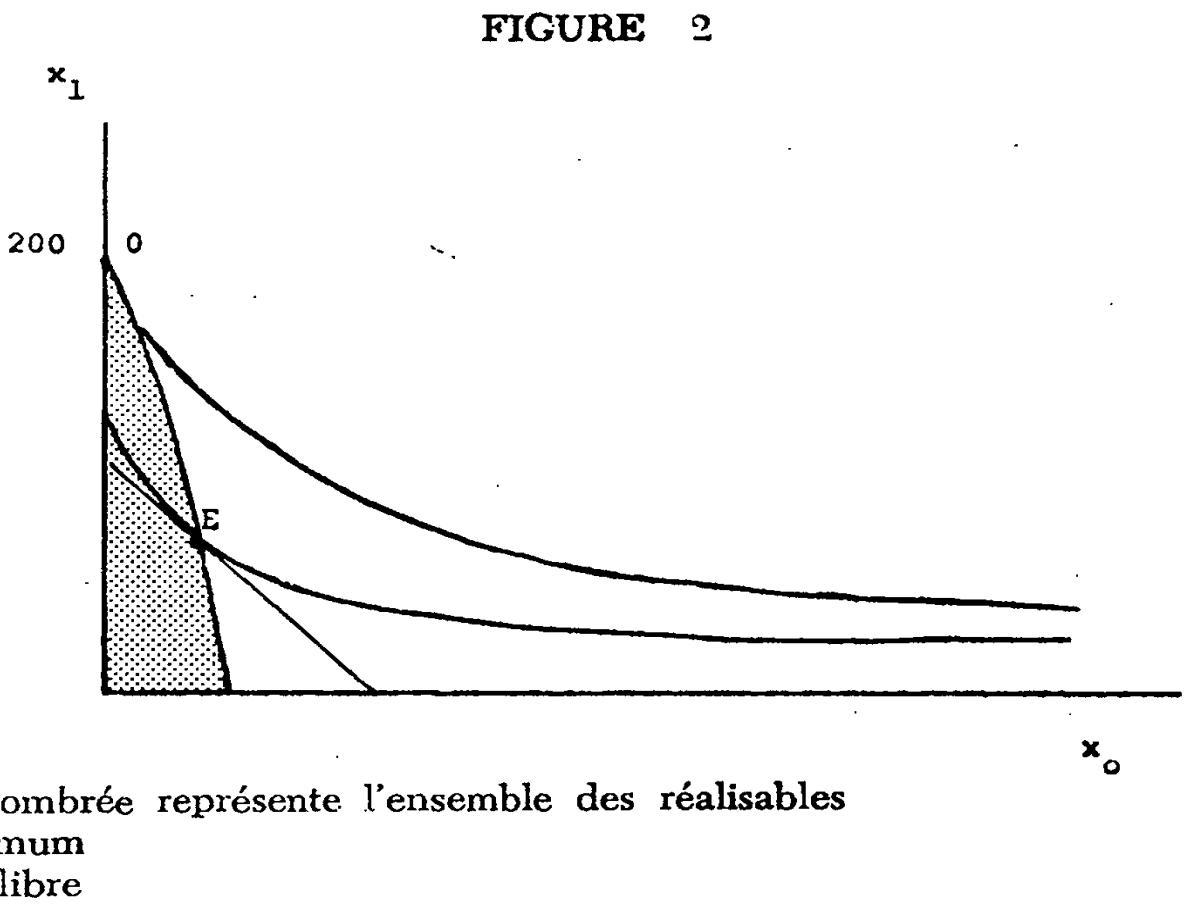

Liexemple que nous venons de donner pourrait suggérer que l'équilibre est globalement inefficace mais localement efficace. Il n'en est rien.

Hyротнѐse 5. $\mathrm{V}(x)$ est strictement quasi concave et différentiable.

Proposirron 4.2. Sous H1-H5 l'équilibre est en général localement inefficace.

Démonstration. Soit $(\bar{q}, \bar{x})$ un équilibre uniforme fort qui est intérieur. La contrainte de ressources s'écrit :

$$
(1+\rho)\left(x_{0}-w_{0}\right)+\sum_{i=1}^{\infty} \Pi_{s}\left(x_{0}\right)\left(x_{0}-w_{0}\right)=0
$$


Nous difféıcncions (4.6) pour obtenir les variations possibles autou. de l'équilibre.

$$
(1+\rho) d x_{0}+\sum_{s=1}^{S} \Pi_{s}\left(x_{0}\right) d x_{s}+\left[\sum_{s=1}^{S} \frac{d \Pi_{s}\left(x_{0}\right)}{d x_{0}} \cdot\left(x_{s}-w_{s}\right)\right] d x_{0}=0
$$

Les conditions du premier ordre de l'équilibre sont obtenues ' partir du programme suivant :

$$
\operatorname{Max} \sum_{s=1}^{s} \Pi_{g}\left(x_{0}\right) U_{s}\left(x_{0}, x_{8}\right)
$$

$$
\text { T.Q. } \quad x_{0}+\sum_{s=1}^{s} q_{s} x_{s} \leqq w_{0}+\sum_{s=1}^{\dot{S}} q_{s} w_{x}
$$

$$
\sum_{d=1}^{S}\left\{\frac{d \Pi_{s}\left(x_{0}\right)}{d x_{0}} U_{s}\left(x_{0}, x_{s}\right)+\Pi_{s}\left(x_{0}\right) \frac{\partial U_{s}}{\partial x_{0}}\left(x_{0}, x_{s}\right)\right\}=\lambda
$$

$$
\begin{aligned}
I_{s}\left(x_{0}\right) \frac{\partial U}{\partial x_{s}}\left(x_{0}, x_{s}\right)=\lambda a_{s} & =\lambda \Pi_{s}\left(x_{0}\right) /(1+\rho) \quad \text { à l'équili ure } \\
& =1, \ldots, \mathrm{S}
\end{aligned}
$$

Pour savoir si nous pouvons améliorer localement l'allocation d'équilibre, nous différencions la fonction d'utilité à l'équilibre :

$$
\begin{gathered}
\sum_{s=1}\left[\frac{d \Pi_{s}\left(x_{0}\right)}{d x_{0}} U_{s}\left(x_{0}, x_{s}\right)+\Pi_{s}\left(x_{0}\right) \frac{\partial U_{s}}{\partial x_{0}}\left(x_{0}, x_{s}\right)\right] d x_{0} \\
+\sum_{s=1}^{S} \Pi_{s}\left(x_{0}\right) \frac{\partial U_{s}}{\partial x_{s}}\left(x_{0}, x_{s}\right) d x_{s}
\end{gathered}
$$

Nous substituons (4.8) dans (4.9).

$$
(1+\rho) \lambda d x_{0}+\lambda \sum_{j=1}^{S} \Pi_{s}\left(x_{0}\right) d x_{0}
$$

D’après (4.7) :

$$
-\lambda\left[\sum_{s=1}^{8} \frac{d \Pi_{s}\left(x_{0}\right)}{d x_{0}}\left(x_{s}-w_{s}\right)\right] d x_{0}
$$

Nous savons que $\lambda$, utilité marginale du revenu, est positive. Selun le signe de $\prod_{s=1}^{\mathbf{s}} \frac{d \Pi_{s}\left(x_{0}\right)}{d x_{0}}\left(x_{s}-w_{s}\right)$, le signe de la variation $d x_{0}$ peut être choisi de sorte que (4.11) soit négatif. L'équilibre est donc localement inefficace. Notons deux cas particuliers : si $\Pi$, est constant. 
$s=1, \ldots, \mathrm{S}$, l'expression (4.11) disparaît. De même, si $x_{s}-w_{s}=a$ pour tout $s$,

$$
\begin{gathered}
\sum_{s=1}^{\mathbf{S}} \frac{d \Pi_{s}\left(x_{0}\right)}{d x_{0}} \cdot\left(x_{s}-w_{s}\right)=a \sum_{s=1}^{\mathbf{S}} \frac{d \Pi_{s}\left(x_{0}\right)}{d x_{0}}=0 \\
\text { puisque } \sum_{s=1}^{\mathbf{S}} \Pi_{s}\left(x_{0}\right)=1 \text { pour tout } x_{0}
\end{gathered}
$$

Q.E.D.

L'analogie suivante en termes d'effets externes permet de se faire une idée plus intuitive du résultat d'inefficacité.

La compagnie d'assurance peut être considérée comme un producteur dont linput est une unité de bien dans la première période et l'output, un produit joint, constitué de $\alpha_{s}$ unités de bien consommable seulement par des consommateurs âgés dans l'état $s, s=1, \ldots, \mathrm{S}$. La fonction de production fictive est donnée par la contrainte budgétaire de la compagnie d'assurance :

$$
\left\{\begin{array}{l}
\alpha_{s}=\frac{1+p}{\Pi_{s}\left(x_{0}\right)} \\
\sum_{s=1}^{s} \frac{1}{\alpha_{s}}=1+\rho
\end{array}\right.
$$

où $x_{0}$ est la consommation individuelle des jeunes agents. Le comportement de ces agents influence les coefficients techniques du producteur « compagnie d'assurance». Il y a donc externalité de consommation sur la production?. Il est rationnel pour un consommateur de négliger son impact sur ces coefficients techniques en raison du grand nombre d'agents.

Puisque l'équilibre concurrentiel est inefficace, il est bon de s'interroger sur. l'existence de politiques économiques qui permettent de rétablir l'optimalité paretienne. C'est l'objet des deux sections suivantes.

7. Il s'agit d'une externalité d'atmosphère (cf. Meade [17]) pour laquelle il est bien connu qu'un système de taxe optimal non personnalisé existe en général. 


\section{SOLUTIONS DE PREMIER RANG \\ A L'INEFFICACITE DE L'EQUILIBRE CONCURRENTIEL}

\section{A. Prime d'assurance variable}

L'inefficacité de l'équilibre concurrentiel vient de la dépendance des distributions de probabilité des états vis-à-vis de variables manipulées par les agents économiques; une solution simple est bien sûı d'avoir une prime d'assurance qui prend en compte l'hétérogénéité des risques.

Proposition 5.1. Si la prime d'assurance est définie par $q_{s}=\frac{\Pi_{s}\left(x_{0}\right)}{1+\rho}$, $s=1, \ldots, \mathrm{S}$, sous $\mathrm{H} 1-\mathrm{H} 4$, il existe un équilibre uniforme fort qui est Pareto efficace.

Démonstration. Un consommateur-type a maintenant le programme suivant :

$$
\begin{gathered}
\operatorname{Max} \mathrm{V}(x) \\
\text { T.Q. }(1+\rho)\left(x_{0}-w_{0}\right)+\sum_{s=1}^{S} \Pi_{s}\left(x_{0}\right)\left(x_{s}-w_{s}\right) \leqq 0 \\
x_{s} \geq 0 \quad s=0,1, \ldots, \mathrm{S}
\end{gathered}
$$

Il existe une solution puisque $\mathrm{V}(x)$ est continue et l'ensemble budgétaire compact. Toute solution ou toute combinaison convexe de solutions est un équilibre efficace.

Soit $\left[\Delta^{*},\left(\lambda_{8 *}\right),\left(x^{8 *}\right)\right]$ une combinaison convexe de solutions du programme (6.1).

Supposons qu'il existe une allocation réalisable et préférée, $\left[\Delta,\left(\lambda_{\varepsilon}\right)\right.$, $\left(x^{\natural}\right)$ ], i.e., telle qu'il existe $\delta^{\prime} \in \Delta$ avec $U\left(x^{8 \prime}\right)>U\left(x^{8 *}\right)$ et $U\left(x^{8}\right) \supseteq U\left(x^{z *}\right)$ pour tout autre $\delta$.

D'après la contrainte de possibilité,

- ou bien

a) $(1+\rho)\left(x_{0}^{8}-w_{0}\right)+\sum_{s=1}^{s} \Pi_{s}\left(x_{0}^{8}\right)\left(x_{s}^{s}-w_{s}\right) \leqq 0$ pour tout $\delta \in \Delta$ 
- ou bien

b) il existe $\delta^{\prime \prime} \in \Delta:(1+\rho)\left(x^{8 / \prime_{0}}-w_{0}\right)+\sum_{s=1}^{S} \Pi_{s}\left(x^{8 \prime \prime}\right)\left(x^{8 \prime \prime}{ }_{s}-w_{8}\right)<0$

Dans le cas $a$ ), $x^{\delta}$ est réalisable pour tout $\delta \in \Delta$, de sorte que $U\left(x^{\varepsilon}\right) \leqq U\left(x^{\delta *}\right)$ pour tout $\delta \in \Delta$, une contradiction.

Dans le cas $b)$, par $\mathrm{H} 2, \mathrm{U}\left(x^{\prime \prime \prime}\right)<\mathrm{U}\left(x^{8 *}\right)$, une contradiction.

Q.E.D.

La politique de prime variable définie par la proposition 5.1 conduit à une allocation efficace des ressources, parce qu'elle oblige chaque agent à prendre en compte, de façon appropriée, non seulement l'ettet de l'achat de $x_{0}$ sur sa distribution de probabilité, mais aussi l'eflet sur les ressources qui résulte d'une redistribution des agents entre les différents états (qui est ici synonyme de l'eftet sur les prix des assurances).

Les difficultés «informationnelles 》 de cette solution sont considérables. La compagnie d'assurance doit connaître la forme des fonctions $\Pi_{s}(),. s=1, \ldots, S$, mais aussi elle doit connaître le montant exact $x_{0}$ pour chaque agent. Notons toutefois que la prime est linéaire par rapport à la quantité d'assurance achetée et donc compatible avec une certaine concurrence entre les compagnies d'assurance.

\section{B. Taxation}

L'analogie avec les externalités, évoquée à la section 3 , suggère la possibilité de taxation.

Proposimion 5.2. Sous H1-H5, il existe un système de taxes, qui soutient une allocation efficace :

$$
\left\{\begin{array}{c}
t_{0}=\left.\sum_{s=1}^{s} \frac{d \Pi_{s}}{d x_{0}}\right|_{x_{0}=x_{0}^{*}\left(x_{s}-w_{s}\right)} \\
t_{s}=0 \quad s=1, \ldots, \mathrm{S}
\end{array}\right.
$$

où $t_{0}$ est le taux de taxation sur la consommation des jeunes agents et $t_{s}$ le taux de taxation sur la consommation des agents âgés dans l'état $s=1, \ldots, \mathrm{S}$.

Démonstration : Soit $x *$ une allocation (intérieure) efficace, solution de : 


$$
\begin{gathered}
\operatorname{Max} \mathrm{V}(x) \\
\mathrm{T} \cdot \mathrm{Q} \cdot \quad(1+\rho) \cdot\left(x_{0}-w_{0}\right)+\sum_{s=1}^{\mathrm{s}} \Pi_{s}\left(x_{0}\right)\left(x_{s}-w_{s}\right)=0
\end{gathered}
$$

Les conditions du premier ordre s'écrivent :

$$
\begin{aligned}
& \sum_{s=1}^{S}\left[\frac{d \Pi_{s}\left(x_{0}\right)}{d x_{0}} U_{s}\left(x_{0}, x_{s}\right)+\Pi_{s}\left(x_{0}\right) \frac{\partial U_{s}}{\partial x_{0}}\left(x_{0}, x_{s}\right)\right]= \\
& \quad \mu\left[1+\rho+\sum_{s=1}^{S} \frac{d \Pi_{s}\left(x_{0}\right)}{d x_{0}}\left(x_{s}-w_{s}\right)\right] \\
& \Pi_{s}\left(x_{0}\right) \cdot \frac{\partial U_{s}}{\partial x_{s}}\left(x_{0}, x_{s}\right)=\mu \Pi_{s}\left(x_{0}\right) \quad s=1, \ldots, S
\end{aligned}
$$

D'autre part, le problème d'optimation d'un consommateur dans une économie avec taxation s'écrit :

$$
\operatorname{Max} V(x)
$$

$$
\begin{array}{ll}
\text { T.Q. } & x_{0}\left(1+t_{n}\right) \leqq w_{n}-\sum_{s=1}^{s} z_{s} q_{s} \\
& x_{s}\left(1+t_{s}\right) \leqq w_{3}+z_{s} \quad s=1, \ldots, \mathrm{S}
\end{array}
$$

Les conditions du premier ordre sont :

$$
\begin{gathered}
\sum_{s=1}^{S}\left[\frac{d \Pi_{s}}{d x_{0}} U_{s}\left(x_{0}, x_{s}\right)+\Pi_{s}\left(x_{0}\right) \frac{\partial U_{s}}{\partial x_{0}}\left(x_{0}, x_{s}\right)\right]=\mu\left(1+t_{0}\right) \\
\Pi_{s}\left(x_{0}\right) \frac{\partial U_{s}}{\partial x_{s}}\left(x_{0}, x_{s}\right)=\mu q_{s}\left(1+t_{s}\right)
\end{gathered}
$$

avec à l'équilibre $q_{s}=\frac{\Pi_{s}\left(x_{0}\right)}{1+\rho}$

L'identification des deux ensembles de conditions du premier ordre donne le résultat.

Q.E.D.

Notons que ce système de taxes a des traits particuliers. Les jeunes agents payent des taxes différentes de celles payées par les vieux. S'il y avait plusieurs types d'agents, chaque type aurait son système de taxes, d'où l'hypothèse implicite que les vendeurs sont capables et désireux de taxer différemment des agents différents. 


\section{FIGURE 3}

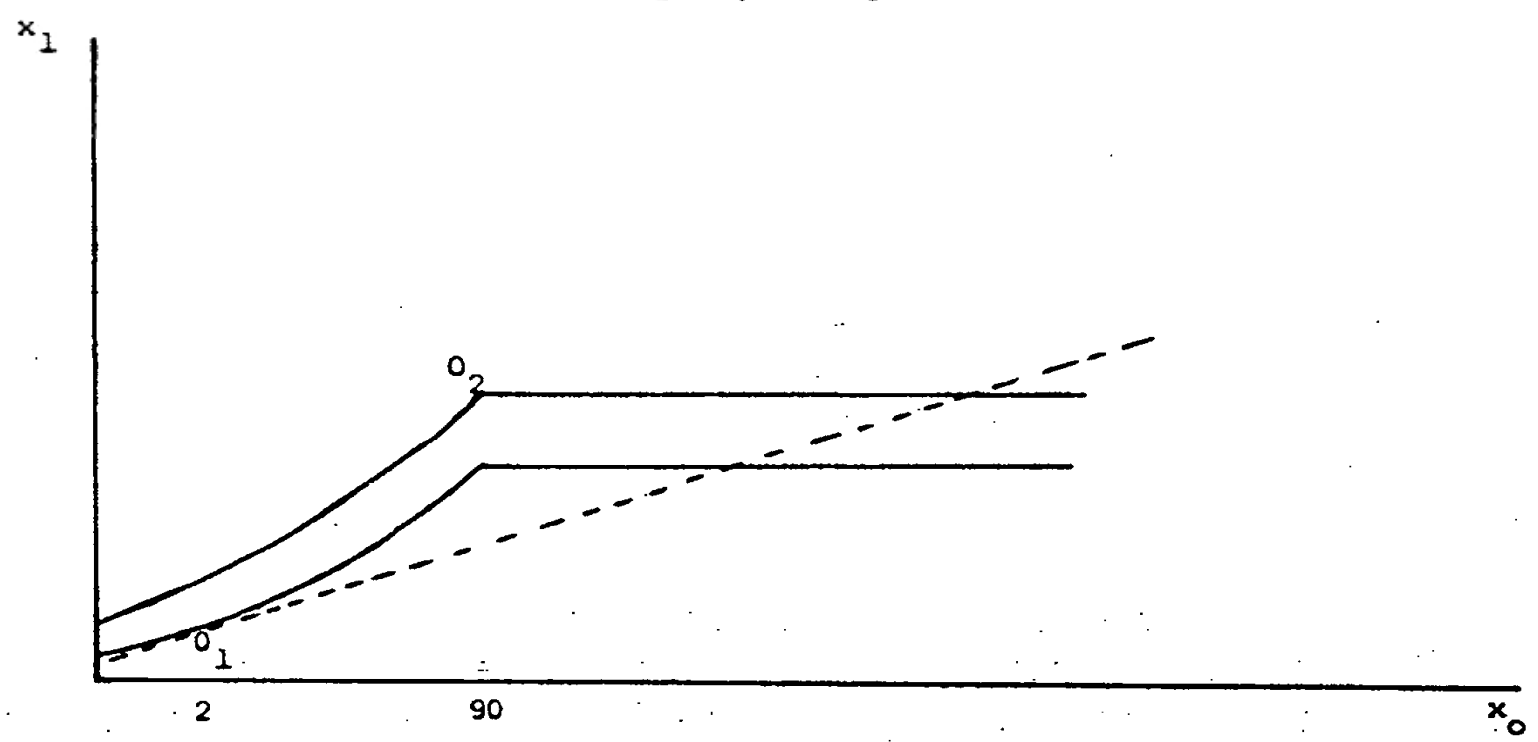

L'optimum $0_{1}$ (pour $w_{2}=106$ ) est obtenu avec $x_{0}=2$. Il est clair sur la figure qu'il ne peut être supporté globalement par un système de taxes, bien qu'il le soit localement. Si $w_{2}=106 / 8-10$, l'optimum $0_{2}$, obtenu avec $x_{0}=90$, ne peut même pas être supporté localement.

Quand V $(\boldsymbol{x})$ n'est pas quasi concave, un système de taxes efficace peut encore exister ${ }^{8}$ (comme dans un exemple du type de celui de la section 3 quand un équilibre existe). Cependant, en général, il ne sera pas possible de supporter le point efficace, même localement par un système de prix. Les non-convexités du problème empêchent donc la solution de taxation. Nous donnons ci-après un exemple où la taxation est impossible.

Soit

$$
\begin{array}{cc}
S=2, U_{1}\left(x_{0}, x_{1}\right)=x_{1}, U_{2}\left(x_{0}, x_{2}\right)=0 & \rho=0 \\
w_{0}=10 . \quad w_{1}=10, \quad w_{2}=106 \\
\Pi_{1}\left(x_{0}\right)=9\left(x_{0}+10\right)^{-2} & 0 \leqq x_{0} \leqq 90 \\
=910^{-4} & x_{0} \geqq 90
\end{array}
$$

8. Lorsqu'il est possible de supporter globalement le point efficace mais qu'il existe des nonconvexités, il est très difficile de trouver les taxes optimales et les processus itératifs de recherche de taxes optimales peuvent converger vers des solutions non optimales. 


\section{SOLUTIONS DE SECOND RANG}

Les politiques économiques proposées à la section 5 sont extrêmement couteuses en termes d'information. En particulier, la solution d'une prime d'assurance variable est très séduisante, mais les coûts de contrôle ex ante du niveau des variables (appelées «soins 》 dans cette section) qui influencent les probabilités sont souvent prohibitifs. Une autre approche est attrayante. Est-il possible de relier les primes d'assurance à des variables plus facilement identifiables et d'améliorer ainsi l'allocation des ressources par rapport à l'équilibre concurrentiel sans toutefois atteindre un optimum de Pareto ? Deux telles solutions sont étudiées ci-après.

A. Pauly [21] a suggéré récemment de relier la prime à la quantité totale d'assurance achetée d'une façon non linéaire, tout en conservant la contrainte de profits nuls pour la compagnie d'assurance. L'idée est d'utiliser la quantité de co-assurance comme signal d'auto-protection. Notons que les coûts à supporter pour trouver une fonction prime d'équilibre sont certainement d'un autre degré de grandeur que les coûts nécessaires pour trouver un prix d'équilibre. Etudions cette proposition en modifiant quelque peu notre modèle.

Soit $\tilde{w}$ le niveau de revenu de la $2^{\mathrm{e}}$ période par rapport auquel sont définies les pertes $l_{s}=\bar{w}-w_{s}, s=1, \ldots, \mathrm{S}$; nous supposons que les valeurs des paramètres sont telles que les agents veulent s'assurer positivement pour tous les états de la deuxième période (cette hypothèse simplificatrice nous permet d'utiliser encore ce modèle particulièrement simple). Soit $\alpha_{s}$ la proportion de la perte $l_{s}$ pour laquelle l'agent désire s'assurer et soit $q_{s}\left(\alpha_{s}\right)$ le prix unitaire d'assurance pour l'état $s$ si l'agent assure une proportion $\alpha_{s}$. de sa perte. ( $\rho=0$ par simplicité).

Propositron 6.1. Considérons un optimum de Pareto; sous des hypothèses appropriées de concavité, il existe une infinité de fonctions $q_{s}\left(\alpha_{s}\right)$ qui permettent de décentraliser l'optimum; elles sont caractérisées par leur valeur à l'équilibre et la valeur de leur dérivée à l'équilibre. 
Démonstration. Le programme d'un consommateur face à une fonction prime $q_{s}\left(\alpha_{s}\right)$ s'écrit :

$$
\begin{aligned}
& \operatorname{Max} \sum_{s=1}^{S} \Pi_{s}\left(x_{0}\right) U_{s}\left(x_{0}, x_{R}\right) \\
& \text { S.T. } \quad x_{0}=w_{0}-\sum_{s=1}^{S} q_{s},\left(\alpha_{s}\right) 1_{s}, \alpha_{s}, \\
& x_{s}=\bar{u}-\left(1-x_{s}\right) 1_{s} \quad s=1, \ldots, \mathrm{S} \\
& x_{z} \geq 0 \quad s=0,1, \ldots, \mathrm{S} \\
& \alpha, \geq 0 \quad s=1, \ldots, \mathrm{S}
\end{aligned}
$$

Les conditions du premier ordre d'un maximum intérieur sont :

$$
\begin{gathered}
{\left[\alpha_{s} \frac{d q_{s}}{d \alpha_{s}}\left(\alpha_{s}\right)+q_{s}\left(\alpha_{s}\right)\right] \cdot \sum_{s=1}^{S}\left[U_{s}, \frac{d \Pi_{s}}{d x_{0}}+\Pi_{s}, \frac{\partial U_{s \prime}}{\partial x_{0}}\right]=\Pi_{s}\left(x_{0}\right) \frac{\partial U_{s}}{\partial x_{s}}} \\
s=1, \ldots, \mathrm{s}
\end{gathered}
$$

Les optima sont caractérisés par le programme suivant :

$$
\begin{array}{cc} 
& \operatorname{Max} \sum_{s=1}^{S} \Pi_{s}\left(x_{0}\right) U_{s}\left(x_{0}, x_{k}\right) \\
\text { S.T. } & x_{0}+\sum_{s=1}^{S} \Pi_{s}\left(x_{0}\right)\left(x_{s}+I_{s}\right)=w_{0}+\bar{u} \\
& x_{s} \geq 0 \quad s=0, \ldots, S
\end{array}
$$

Les conditions du premier ordre d'un optimum intérieur sont :

$$
\sum_{s=1}^{S}\left[U_{s,} \frac{d \Pi_{s^{\prime}}}{d x_{0}}+\Pi_{s^{\prime}} \frac{\partial U_{s \prime}}{\partial x_{0}}\right]=\frac{\partial U_{s}}{\partial x_{s}}\left[1+\sum_{s^{\prime}=1}^{S} \frac{d \Pi_{s \prime}}{d x_{0}}\left(\bar{w}+\alpha_{s}, 1_{s},\right)\right]
$$

Ces conditions se simplifient quelque peu car

$$
\sum_{s=1}^{s} \frac{d \Pi_{s \prime}}{d x_{0}} \bar{w}=\bar{w} \sum_{s,=1}^{s} \frac{d \Pi_{s \prime}}{d x_{0}}=0 \text { puisque } \sum_{s ;=1}^{s} \Pi_{s},\left(x_{0}\right)=1
$$

L'identification des deux ensembles de conditions du premier ordre donne :

$$
\frac{\Pi_{s}\left(x_{n}\right)}{\alpha_{s} \frac{d q_{s}}{d \alpha_{s}}\left(\alpha_{s}\right)+q_{s}\left(\alpha_{s}\right)}=1+\sum_{s=1}^{s} \frac{d \Pi_{s}}{d x_{0}} \alpha_{s,} 1_{s}
$$


L'équilibre budgétaire de la compagnie d'assurance exige :

$$
\alpha_{s} 1_{s} \Pi_{s}\left(x_{0}\right)=q_{s}\left(\alpha_{s}\right) \alpha_{s} 1_{s}
$$

ou

$$
\Pi_{s}\left(x_{0}\right)=q_{s}\left(\alpha_{s}\right)
$$

Si nous portons cette contrainte dans (6.3), nous obtenons une condition que doit vérifier l'élasticité de la fonction $q_{s}($.$) à l'équilibre :$

$$
e_{\mathrm{q}}\left(\alpha_{s}\right)-\frac{d q_{s}}{d \alpha_{s}} \cdot \frac{\alpha_{s}}{q_{s}}=\frac{a}{1+a}
$$

avec

$$
a=\sum_{s=1}^{s} \frac{d \Pi_{s,}}{d x_{0}} \alpha_{s}, 1_{s^{\prime}}
$$

Par conséquent, les conditions du premier ordre imposent à la tarification les deux contraintes à l'équilibre :

$$
\begin{array}{ll}
q_{s}\left(\alpha_{s}^{*}\right)=\Pi_{s}\left(x_{v} j\right. & s=1, \ldots, \mathrm{S} \\
e_{q}\left(\alpha_{s}^{*}\right)=\frac{a^{*}}{1+a^{*}} & s=1, \ldots, \mathrm{S}
\end{array}
$$

Q.E.D.

Notons que lorsque $\Pi_{s}($.$) ne dépend pas de x_{0}, s=1, \ldots, \mathrm{S}$, nous retrouvons que $q_{s}$ doit être indépendant de $\alpha_{s}$.

Il y a deux types de difficultés dans une telle solution. La première est technique et concerne la multiplicité d'équilibres que l'on peut obtenir avec des fonctions $q_{s}$ données. Certains de ces équilibres ne sont pas des optima de Pareto même si les fonctions $q_{s}($.$) satisfont les$ conditions données ci-dessus. On peut espérer que la concurrence des compagnies d'assurance éliminera de tels équilibres. La deuxième difficulté concerne les exigences informationnelles de cette solution. Il est vrai qu'en principe, à l'équilibre, la compagnie d'assurance n'a pas besoin de connaître $x_{0}$. Cependant, pour parvenir à un tel équilibre cette connaissance semble indispensable. De plus, les compagnies doivent se transmettre de linformation pour savoir quels contrats l'agent a déjà signé et pour pouvoir lui appliquer la prime non linéaire $q_{s}($.$) .$ Si la prime $q_{s}($.$) satisfait la condition d'équilibre budgétaire mais ne$ satisfait pas la condition d'élasticité, l'équilibre n'est pas un optimum de Pareto. Une question non résolue est : quelles conditions doivent satisfaire $q_{s}(),. s=1, \ldots, \mathrm{S}$, pour que cet équilibre à primes non linéaires soit préférable à l'équilibre concurrentiel défini à la section 2 ? 
B. Nous suggérons dans cette sous-section une autre solution basée sur la simple remarque suivante. Les états qui donnent lieu à assurance et qui correspondent en général à des «accidents» ont de faibles probabilités, de sorte que seule une faible proportion de la population est en fait dans ces états. L'idée est donc de remplacer le contrôle ex ante des soins sur tous les agents, par un contrôle ex post sur les seuls agents ayant des accidents. Quand un consommateur achète une quantité d'assurance, il annonce à la compagnie la quantité de soins qu'il va prendre dans la première période. Les primes sont calculées d'après cette déclaration. Après l'accident, la quantité de soins annoncée fait l'objet d'une enquête. Nous supposons qu'il existe une Cour de justice qui est chargée de juger l'exactitude de la déclaration de l'assuré. S'il est établi par la Cour qu’il a trompé l'assurance, celle-ci n’a pas à payer les indemnités spécifiées dans le contrat. Pour éviter partiellement les coûts de transaction élevés du système judiciaire, la compagnie d'assurance peut conduire sa propre enquête et n'aller devant la Cour que si elle est en désaccord avec son client (ou pense qu'elle peut gagner le procès). Car il est évident que la Cour ne sera pas parfaite, en particulier à cause des pertes d'information dues au décalage temporel entre la date de l'action et la date de l'enquête. Dans de nombreux cas, il est possible néanmoins de réaliser un contrôle ex post précis lorsque, par exemple, il s'agit de l'existence d'un procédé anti-feu. Dans d'autres cas, cela est plus difficile ou même impossible.

Soit $x_{0}$ le niveau de soins réel et $\tilde{\boldsymbol{x}}_{0}$ le niveau de soins annoncé.

La Cour de justice est une variable aléatoire qui prend les valeurs un et zéro (signifiant non coupable de négligence ou coupable) avec les probabilités $\Psi\left(x_{0}-\tilde{x}_{0}\right)$ et $1-\Psi\left(x_{0}-\tilde{x}_{0}\right)$ qui dépendent de l'écart entre les valeurs réelle et annoncée de soins.

Une décision de la Cour est une valeur de cette variable aléatoire.

$\Pi_{s}($.$) est maintenant supposé décroissante, s=2, \ldots, \mathrm{S}$.

Si $\Psi(y)=1$ pour $y \geqslant 0$ et $\Psi(y)=0$ pour $y<0$ la Cour est infaillible.

Soit $\widetilde{\Pi}\left(\tilde{x}_{0}\right)$ la fonction prime dépendant du niveau de soins annoncé, qui est proposée par la compagnie d'assurance.

Dans les notations des sections précédentes, le problème d'optimation du consommateur peut s'écrire maintenant :

$$
\operatorname{Max} \sum_{s=1}^{S} \Pi_{s}\left(x_{0}\right)\left[\Psi\left(x_{0}-\tilde{x}_{0}\right) U_{s}\left(x_{n}, x_{s}\right)+\left(1-\Psi\left(x_{0}-\tilde{x}_{0}\right)\right) U_{s}\left(x_{0}, w_{s}\right)\right]
$$




$$
\begin{array}{rlrl}
\text { T.Q. } \quad x_{0} & =w_{0}-\sum_{s=2}^{\mathrm{s}} \widetilde{\Pi}_{s}\left(\tilde{x}_{0}\right) z_{s} & \\
x_{s} & =w_{s}+z_{s} & s & =1, \ldots, \mathrm{S} \\
x_{s} & \geqq 0 & s=0,1, \ldots, \mathrm{S}
\end{array}
$$

où $\Psi\left(x_{0}-\tilde{x}_{0}\right)$ est l'anticipation de la Cour par le consommateur. Une fonction prime d'équilibre sera une fonction $\tilde{\Pi}_{s}\left(\tilde{x}_{0}\right), s=1, \ldots, S$, compatible avec des profits nuls pour la compagnie d'assurance.

On peut alors démontrer les propositions suivantes (voir [14] pour un énoncé rigoureux et des démonstrations).

Proposition 6.2. Sous des hypothèses de concavité sur l'espérance mathématique de l'utilité, il existe un équilibre.

Propositron 6.3. Si la Cour est infaillible, l'équilibre est un optimum de Pareto.

Proposirion 6.4. Si la Cour est presque infaillible (dans un sens topologique précis), l'équilibre est presque un optimum de Pareto.

Cette dernière proposition est importante car elle démontre l'utilité rle ce système lorsque la Cour n'est pas parfaite.

\section{CONCLUSION}

L'analyse d'équilibre général à laquelle nous nous sommes livrés nous a permis de cerner un certain nombre de difficultés passées inaperçues dans l'analyse partielle du problème d'auto-protection. Tout d'abord les non-convexités fondamentales du problème peuvent conduire parfois à l'impossibilité d'une décentralisation et induisent souvent de délicats problèmes de sélection adverse. L'inefficacité cle l'équilibre concurrentiel nous a conduit à analyser un certain nombre de solutions possibles; dans chaque cas particulier, une analyse des flux d'information disponibles permettra de juger quelle solution est préférable. Souvent, ces différentes solutions seront combinées. Ainsi, on peut imaginer une prime dépendant d'un signal que la compagnie considère lié à $x_{0}$, de la proportion de la perte assurée, de la déclaration de l'agent $x_{0}$, sachant par ailleurs que l'Etat taxe un bien dont la consommation est particulièrement défavorable au risque. 
J'espère que cette note permet de comprendre un peu mieux la rationalité économique de ces diverses mesures. N'oublions pas toutefois les limites de notre modèle, en particulier l'hypothèse que les types d'agents sont identifiables, qui a permis d'isoler une partie du problème créé par l'asymétrie d'information entre consommateurs et compagnies d'assurances.

Le problème que nous avons analysé est en fait immergé dans un problème encore plus complexe d'asymétrie informationnelle.

\section{JEAN-JACQUES LAFFONT}

Maître de conférences

à l'Ecole Polytechnique

\section{REFERENCES}

[1] Akerdor G.A., "The Market for “Lemons" : Quality Uncertainty and the Market Mechanism n, Quarterly Journal of Economics, vol. 84, 1970, pp. 488-500.

[2] Arrow K.J., "The Role of Securities in the Optimal Allocation of RiskBearing ", Review of Economic Studies, vol. 41, 1963-64, pp. 91-96.

[3] Arnow K.J., a Uncertainty and the Welfare Economics of Medical Care ", American Economic Review, vol. 53, 1963, pp. 941-973.

[4] Arrow K.J., " The Economics of Moral Hazard : Further Comment n, American Economic Review, vol. 58, 1968, pp. 537-539.

[5] Arrow K.J., "Optimal Insurance and Generalized Deductibles ", Scand. Actuarial Journal, 1974, pp. 1-42.

[6] Bonch K., The Mathematical Theory of Insurance, Lexington Books, Lexington Massachusetts, 1974.

[7] Debreu G., Theory of Value, Jchn Wiley and Sons, Inc., New York, 1959.

[8] Demsetz H., " Information and Efficiency : Another Viewpoint 》, Journal of Law and Economics, vol. 12, 1959, pp. 1-22.

[9] Ekxh.rch I., BEcker G.S., " Market Insurance, Self-Insurance and Self-Protection ", Journal of Political Economy, vol. 80, 1972, pp. 623-648.

[10] Gourd J.P., « The Expected Utility Hypothesis and the Selection of Optima! Deductibles for a Given Insurance Policy n, Journal of Business, vol. 42, 1969, pp. 143-151. 
[11] Green J., Sheshinskx E., "Competitive Inefficiencies in the Presence of Constrained Transactions ", Journal of Economic Theory, vol. 10, 1975, pp. 343-357.

[12] Hexpman E., Laffont J.J., "On Moral Hazard in General Equilibrium Theory ", Journal of Economic Theory, vol. 10, 1975, p. 1-23.

[13] Larfont J.J., "Insurance with Moral Hazard in a Monetary Economy ", miméo, 1974.

[14] Laffont J.J., "Court Against Moral Hazard ", Miméo, 1974, révisé 1976.

[15] Malinvaud E., "The Allocation of Individual Risks in Large Markets "; Journal of Economic Theory, vol. 5, 1972, pp. 312-328.

[16] Malinvaud E., "Markets for an Exchange Economy with Individual Risks ", Econometrica, vol. 41, 1973, pp. 383-410.

[17] Meade J.E., "External Economies and Diseconomies in a Competitive Situation ", Economic Journal, vol. 62, 1952, pp. 59-67.

[18] Mossin J., "Aspects of Rational Insurance Purchasing ", Journal of Political Economy, vol. 76, 1968, pp. 553-568.

[19] Pashrgian B.P., Schkade L.L., Menefee G.H., " The Selection of an Optimal Deductible for a Given Insurance Policy n, Journal of Business, vol. 39, 1966, pp. 35-44.

[20] PAUxy M.V., "The Economics of Moral Hazard ", American Economic Revieu, vol. 58, 1968. pp. 31-58.

[21] PauLx M.V., "Overinsurance and Public Provision of Insurance : The Roles of Moral Hazard and Adverse Selection ", Quarterly Journal of Economics, vol. 88, 1974, pp. 44-62.

[22] RADNER R., "Competitive Equilibrium Under Uncertainty ", Econometrica, vol. 36,1968 , pp. 31-58.

[23] Rothschicd M., Strglirz J.E., "Equilibrium in Insurance Markets : The Economics of Imperfect Information ", à paraître dans Quarterly Journal of Economics.

[24] Surte V.L., "Optimal Insurance Coverage ", Journal of Political Economy vol. 76,1968 , pp. $68-77$. 